\title{
The association between sedentary behaviour and indicators of stress: a systematic review
}

\author{
Megan Teychenne ${ }^{1 *}$ (D), Lena D. Stephens ${ }^{1}$, Sarah A. Costigan², Dana Lee Olstad ${ }^{3}$, Brendon Stubbs ${ }^{4}$ and
} Anne I. Turner ${ }^{1}$

\begin{abstract}
Background: Emerging evidence shows sedentary behaviour may be associated with mental health outcomes. Yet, the strength of the evidence linking sedentary behaviour and stress is still unclear. This study aimed to synthesise evidence regarding associations between time spent in sedentary behaviour and stress in adults.

Methods: A systematic search was conducted (January 1990 - September 2019). Following PRISMA guidelines, an evaluation of methodological quality, and best-evidence synthesis of associations between time in sedentary behaviour (including sitting time, TV viewing, computer use) and stress were presented. Twenty-six studies reporting on data from $n=72,795$ people (age 18-98y, 62.7\% women) were included.

Results: Across the studies ( $n=2$ strong-, $n=10$ moderate- and $n=14$ weak-quality), there was insufficient evidence that overall time spent in sedentary behaviour and sitting time were associated with stress, particularly when using self-report measures of sedentary behaviour or stress. There was strong evidence of no association between TV viewing, or computer use and stress. Amongst studies using objective measures of sedentary behaviour and/or stress there was also strong evidence of no association.

Conclusion: Although previous research suggested sedentary behaviour may be linked to mental health outcomes such as depression and anxiety, the evidence for an association between various types of sedentary behaviour and stress is limited in quality, and associations are either inconsistent or null. High-quality longitudinal/interventional research is required to confirm findings and determine the direction of associations between different contexts (i.e. purpose) and domains (i.e. leisure, occupational, transport) of sedentary behaviour and stress.
\end{abstract}

Keywords: Sedentary behaviour, Sitting time, Television viewing, Stress, Mental health, Adults

\section{Background}

Psychological stress is a negative emotional state associated with nervousness, tension and/or strain [1, 2], which can be characterised by feelings of worry, fatigue and inability to cope [3]. Stress has been described as the 'modern day hidden epidemic' [2], due to its high and rising prevalence and impact on health worldwide. Approximately $16 \%$ of adults in Sweden [4] and Australia [5], 22\% in Europe [6] and 24\% in the United States (US)

\footnotetext{
* Correspondence: megan.teychenne@deakin.edu.au

${ }^{1}$ Institute for Physical Activity and Nutrition (IPAN), School of Exercise and

Nutrition Sciences, Deakin University, Geelong, Australia

Full list of author information is available at the end of the article
}

[7] report moderate to severe perceived stress. Chronic and/or high levels of stress are associated with increased risk of adverse health conditions, including cardiovascular disease and events [8], depression [9] and long-term disability [10]. Further, psychological stress is linked to reduced workplace productivity and increased absenteeism [11] and is estimated to cost the US USD $\$ 42$ billion [2], the EU-15 €26.47 billion [12], and Australia AUD\$25 billion [13] per year. Given this significant impact, understanding lifestyle factors that may influence (or be influenced by) stress is essential.

There is growing evidence showing that positive lifestyle behaviours such as increased physical activity/exercise [14, 15], a healthy diet [16] and smoking cessation

(C) The Author(s). 2019 Open Access This article is distributed under the terms of the Creative Commons Attribution 4.0 International License (http://creativecommons.org/licenses/by/4.0/), which permits unrestricted use, distribution, and 
[17] are linked to reduced risk of psychological stress, as well as other mental health outcomes such as depression and anxiety symptoms [17-19]. However, less is known regarding the relationship between sedentary behaviour (i.e. sitting or reclining behaviours requiring minimal energy expenditure [20]) and stress. Given that there is an increasing evidence base to suggest that sedentary behaviour (such as computer use, TV viewing, and overall sitting) is linked to poor mental health, specifically depression [21] and anxiety [22], it is plausible that sedentary behaviour may also be linked to stress.

Theoretically, sedentary behaviour could be associated with stress via a number of potential pathways. For example, screen-based sedentary behaviours such as TV viewing, computer or electronic device use (i.e. smartphones, tablets) can induce feelings of addiction [23], 'brain burnout' [5] and/or sleeping problems [24], potentially leading to heightened levels of psychological stress. Further, engaging in such sedentary behaviours may displace time spent in other important activities such as undertaking household or work-related responsibilities, or physical activity, which may then increase feelings of stress [25]. Alternatively, since TV viewing is a popular strategy used by many adults in developed countries to manage stress (e.g. reported by $85 \%$ of respondents from the Stress and Wellbeing in Australia Survey, $n=1521$; and 39\% in the Stress in America Survey, $n=3361$ ) [5], it is possible that some sedentary behaviours may reduce stress. Yet, there is a lack of clarity regarding associations between different types of sedentary behaviour and stress, and no previous review has summarised the evidence to date. Thus, the aim of this review was to investigate associations between time spent in sedentary behaviour and stress in adults.

\section{Methods}

The protocol was registered with PROSPERO: https:// www.crd.york.ac.uk/PROSPERO/ (registration number: CRD42018091235).

\section{Search strategy}

A systematic electronic search was undertaken for articles published from January 1990 to September 2019. Findings are reported according to PRISMA reporting guidelines [26]. Articles prior to 1990 were not included as increases in population sedentary behaviour levels did not begin to be reported until after 1990 with the advent of widespread online technology use [27]. Databases included Medline/Medline Complete, CINAHL Complete, PsychINFO, SPORTDiscus and EMBASE. Full search strings are provided in Additional file 1: Table S1, however, principal search terms encompassed: 1) 'Sedentary behaviour' (sedentary behaviour, screen time, screen-based, television, computer, electronic device, video game, smart phone, sitting, passive transport, and tablet); and 2) 'Stress' (stress, cortisol, adrenocortical/glucocorticoid hormones). Search strings were further limited to peer-reviewed articles written in English. The literature search, and each of the following stages was led by LDS. Duplicates were identified by LDS in two stages. The majority of duplicates were first identified using EndNote's automated 'find duplicates' function. Remaining duplicates were then identified in EndNote by sorting article titles alphabetically before manual checking, then sorting author names alphabetically before again checking manually. After removal of duplicates, article titles were initially screened for inclusion by LDS and MT. Abstracts were then assessed, followed by retrieval of full texts which were read to determine suitability. Reference lists from retrieved full-text articles and authors' own bibliographic libraries were also searched, yielding no additional articles. MT and LDS reviewed all final articles to determine their inclusion in the review.

\section{Study selection criteria}

For the purpose of this review, stress was operationalised as both self-reported (e.g. perceived psychological stress) and objective (e.g. changes in stress hormones such as cortisol) measures of stress. Articles were eligible for inclusion if they: 1) were published in a peer-reviewed journal in English between January 1990 to September 2019 ; 2) examined apparently healthy adults aged $\geq 18$ years (i.e. those not specifically recruited among populations with underlying chronic physical conditions (e.g. diabetes) or mental disorders (e.g. depression)); 3) examined self-reported or objective measures of screen-based sedentary behaviour or other forms of sitting time; 4) assessed self-reported or objective measures of stress; and 5) employed a cross-sectional, longitudinal, direct observation (e.g. where time in sedentary behaviour was collected for one year or one month and averaged), or controlled experimental study design. Qualitative studies and review articles were excluded. Intervention studies that primarily investigated the direct relationship between sedentary behaviour and a measure of stress were eligible to be included; however, studies that reported the effect of an intervention on stress, or sedentary behaviour, independent of one another, were not eligible. Due to potential confounding, articles relating to yoga or meditation for stress relief, or that measured stress in relation to engagement in violent screen-based activities (e.g. viewing violent TV shows, playing violent computer games) were excluded from the review, as were conference abstracts, dissertations, theses, and articles published in non-peer-reviewed journals.

\section{Data extraction}

Data extraction was performed by LDS using a data extraction form that was pilot-tested initially by MT, SAC 
and LDS on three included articles. Key study characteristics were extracted from identified studies included country in which the study took place, study population characteristics (sample size, age, and sex of participants), study design, sedentary behaviour type (e.g. screen-time, sitting time) and measures (e.g. self-reported or objective [i.e. direct observation or device assessed]) of sedentary behaviour, measures of stress (e.g. self-reported or objective measures) and study results in terms of the association between sedentary behaviour and stress.

\section{Methodological quality}

Methodological quality of each study was evaluated using the Effective Public Health Practice Project Quality Assessment Tool for Quantitative Studies - recommended by the Cochrane Public Health Review Group [28]. The tool assesses research studies on selection bias (e.g. representativeness, response rate), study design (e.g. longitudinal, randomized controlled trial), confounders (e.g. controlling for confounders such as sociodemographic characteristics), blinding (e.g. researcher/participant awareness of group allocation), data collection methods (e.g. validity and reliability of measures), withdrawals and dropouts (e.g. reasons, proportion of sample with complete data), intervention integrity (e.g. percent receiving intervention) and analyses (e.g. appropriate statistical analyses for study design). For all studies, each component was given an overall quality score of weak, moderate, or strong, following the established protocol [28]. An overall study rating was assigned to each study as follows: weak (if they received $\geq 2$ weak ratings); moderate (if they received one weak rating); or strong (if they received no weak ratings). The methodological quality of studies was independently assessed by two reviewers (LDS and SAC). Any discrepancies were resolved via discussion.

\section{Best-evidence synthesis}

To enable conclusions on associations between sedentary behaviour and stress on the basis of the methodological quality of studies, a best-evidence synthesis [29] was conducted by two authors (MT and LDS). Due to the heterogeneity in exposure and outcomes, a bestevidence synthesis was selected over a meta-analysis given it would not be meaningful to calculate the average effect as per a meta-analysis $[29,30]$. This method has been used previously in systematic reviews in the area of sedentary behaviour and health outcomes [22, 31, 32]. Adapted from guidelines outlined in previous reviews that applied best-evidence synthesis [22, 31], the evidence was graded as strong, moderate, or insufficient. Consistency was defined on two levels: 1 ) within a study (i.e. $\geq 75 \%$ of results in same direction within a study), to account for multiple modelling; and 2) between studies (i.e. $\geq 75 \%$ of results in same direction across studies examined). Strong evidence was defined as consistent results in $\geq 2$ strong/ moderate quality studies. Moderate evidence was defined as consistent results in one strong/moderate quality study and at least one weak-quality study; or consistent results in $\geq 2$ weak-quality studies. Insufficient evidence was defined as having only one available study or inconsistent results in $\geq 2$ studies. When $\geq 2$ studies were of strong/ moderate methodological quality, those with weak-quality were disregarded in the evidence synthesis [31]. In this manner, evidence was weighted in terms of study design/ methodological quality.

To determine whether associations between time in sedentary behaviour and stress could be explained by the nature of the sedentary behaviour and stress measures, studies were grouped and results analysed firstly on the basis of utilising objective versus self-report measures of stress, and secondly, on the use of objective (i.e. device assessed or direct observation) and self-report measures of sedentary behaviour.

\section{Results}

Literature searching yielded 12,653 articles after duplicates were removed (Fig. 1), which were screened by title. After further screening of abstracts $(n=72)$, and full papers $(n=51)$, a total of 26 studies (reported in 24 papers - note: Anderson et al. (1996) included three studies presented within the one paper [33]) were included in the review. Characteristics of included studies are summarised in Table 1. Sixteen studies employed a cross-sectional design [33-36, 41, 43, 44, 46, 47, 49-51, $53,54,56,57]$ ( $n=72$ to 34,129 participants). Four studies were longitudinal $[33,38,45,55](n=271$ to 11,676$)$, two were controlled clinical trials $[39,48](n=43-231)$, two were pilot interventions $[40,52](n=12$ to 20$)$ and two were direct observation studies (i.e. where time in sedentary behaviour was collected for one year or one month and averaged) [33, 37] ( $n=79$ to 140). The majority of these studies were conducted in Australia $(n=$ $8)$ and the US $(n=8)$. Twenty one of the 26 study samples were comprised of men and women (age 18-98y) [33-41, 43, 44, 47-52, 56, 57], while the remaining five studies were conducted among women only (age 18-65y) $[45,46,53-55]$.

Objective measures of stress were used in $\mathrm{n}=7$ studies, and included salivary $[39-41,52]$ or hair $[43,50,54]$ cortisol, blood pressure (systolic and diastolic) [39] and heart rate [39]. Stress was self-reported using measures including the Life Events Inventory (LEI, $n=3$ ) [33], the Cohen Perceived Stress Scale (PSS, $n=8$ ) [35, 38, 44-47, 50, 53], the Depression, Anxiety and Stress Scale (DASS-21, $n=1$ ) [49], the Perceived Stress Questionnaire for Young Women (PSQYW, $n=1$ ) [55], the affective experience component of the Princeton Affect and Time Survey $(n=1)$ [36], the 


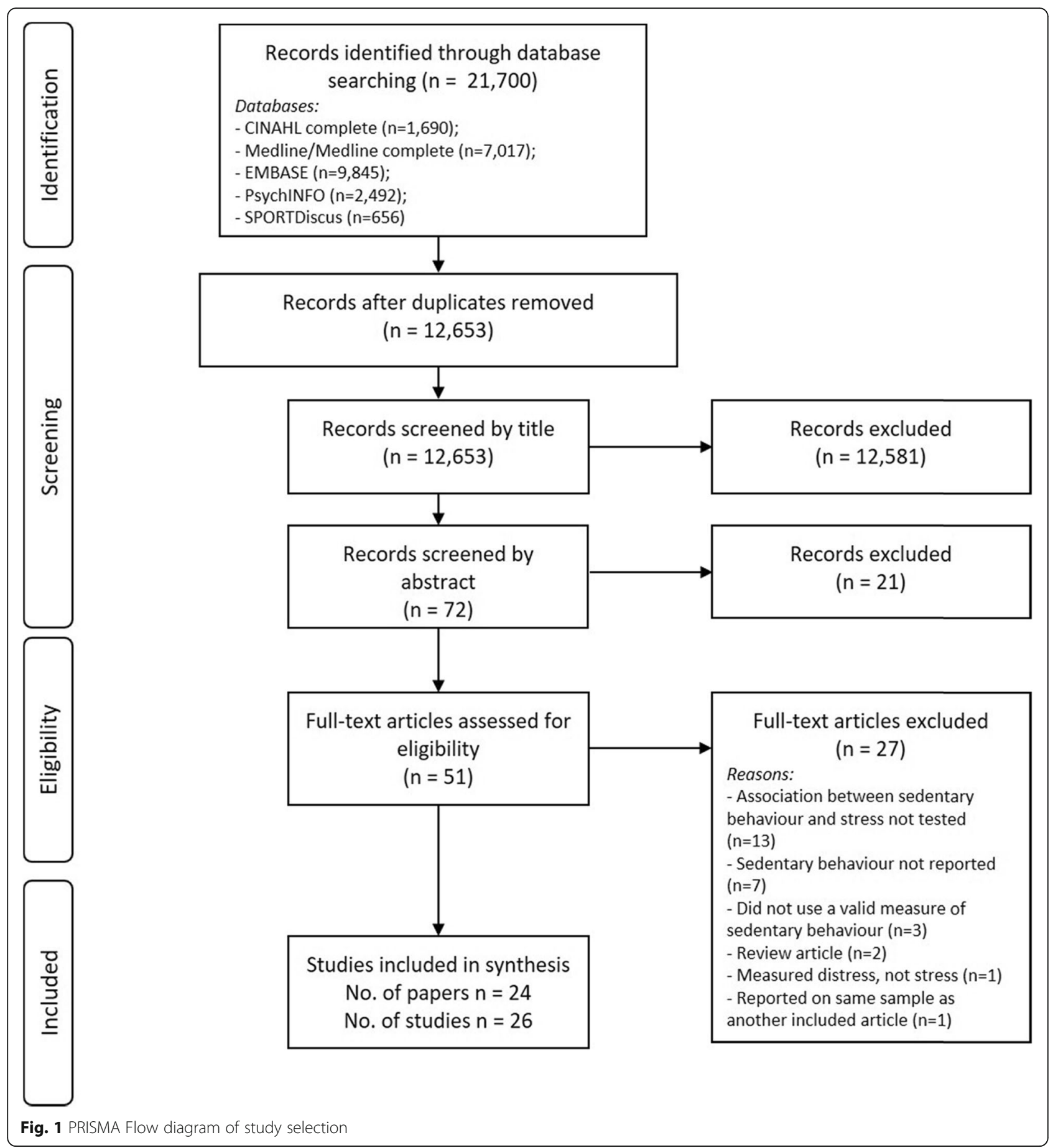

Daily Stress Inventory (DSI, $n=1$ ) [37], the Chronic Burden Scale (CBS, $n=1$ ) [56], the Traumatic Stress Schedule (TSS, $n=1)$ [56], job stressors $(n=1)$ [51], the Health and work Questionnaire $(n=1)$ [48] and the Effort Reward Imbalance Scale (ERIS, $n=1$ ) [57]. One study measured stress using self-report survey items designed specifically for that study [34].

Ten studies measured sedentary behaviour objectively ( $n=7$ utilised accelerometers [37-39, 41, 48, 50, 56]; $n=2$ directly observed participants' sitting time in a laboratory setting $[40,52] ; n=1$ objectively measured TV viewing time via time-lapse video recordings [33]). Selfreport measures of sedentary behaviour were used in 16 studies. Types of sedentary behaviour that were selfreported included: total daily and/or weekly sitting [34, 35, 44, 45, 47, 49, 51, 54-57]; TV viewing time [33, 36, 43, 45, 46, 49, 53, 54]; occupational sitting time [49]; computer use [49, 54]; transport-related 


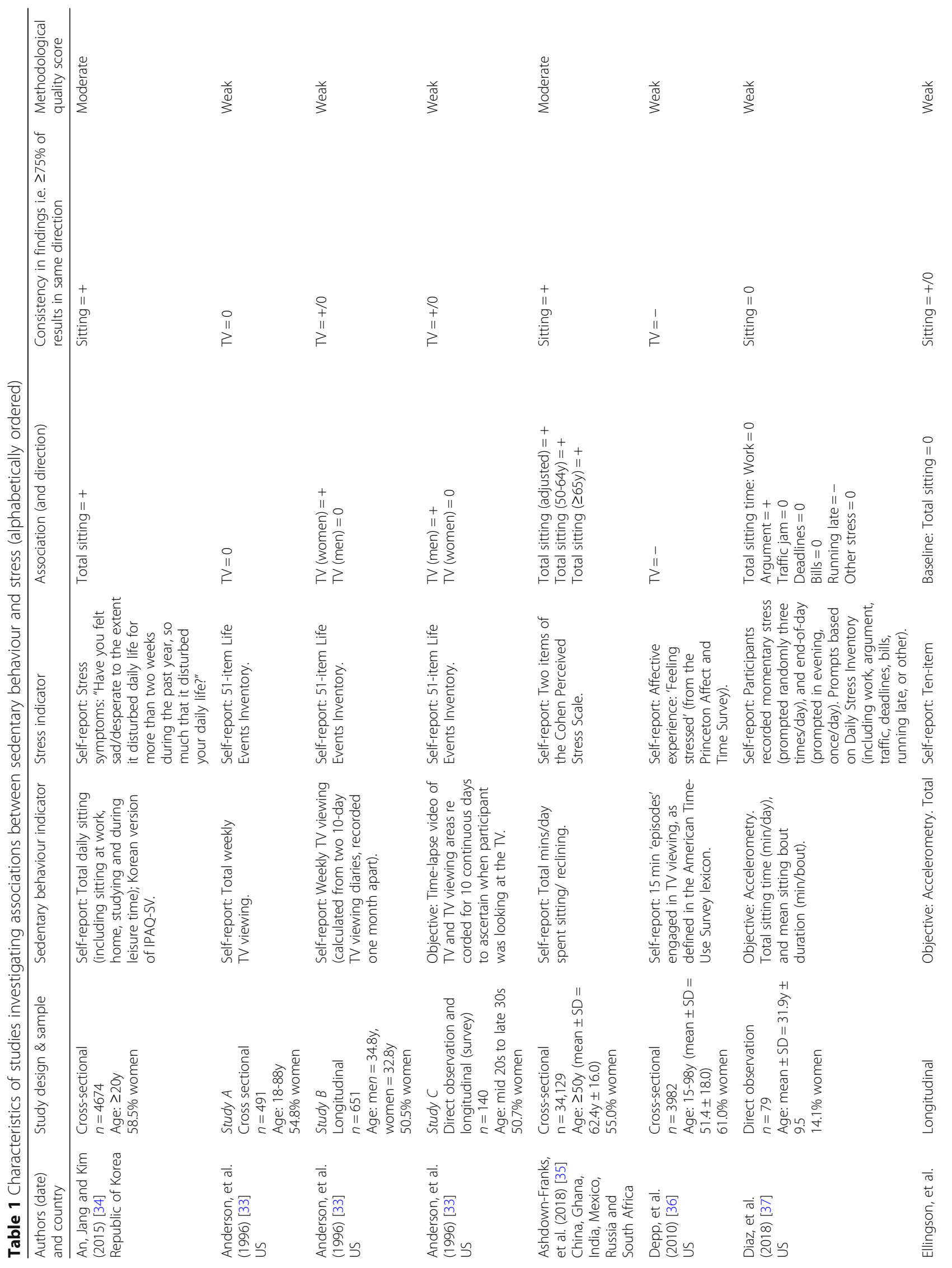




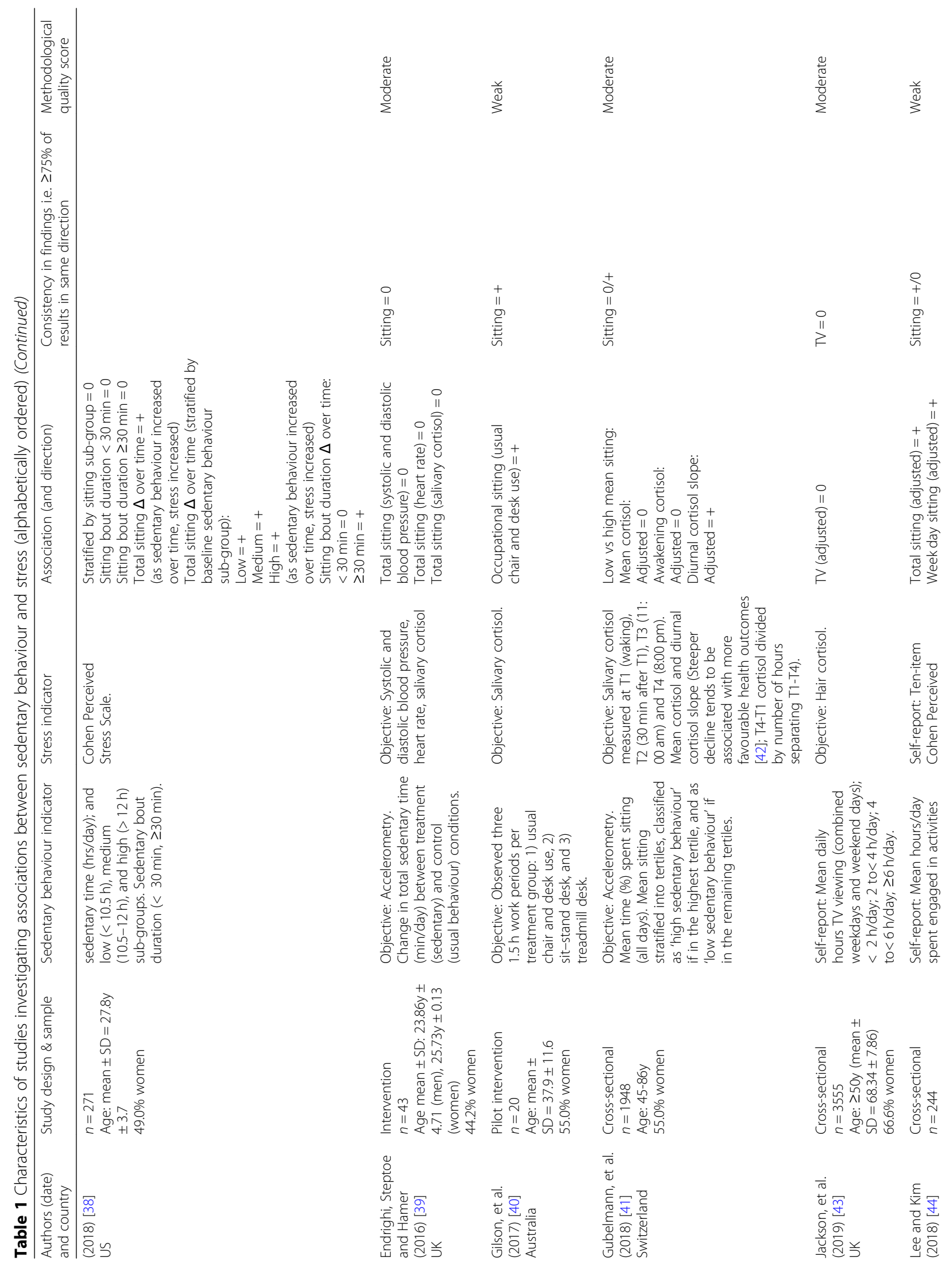




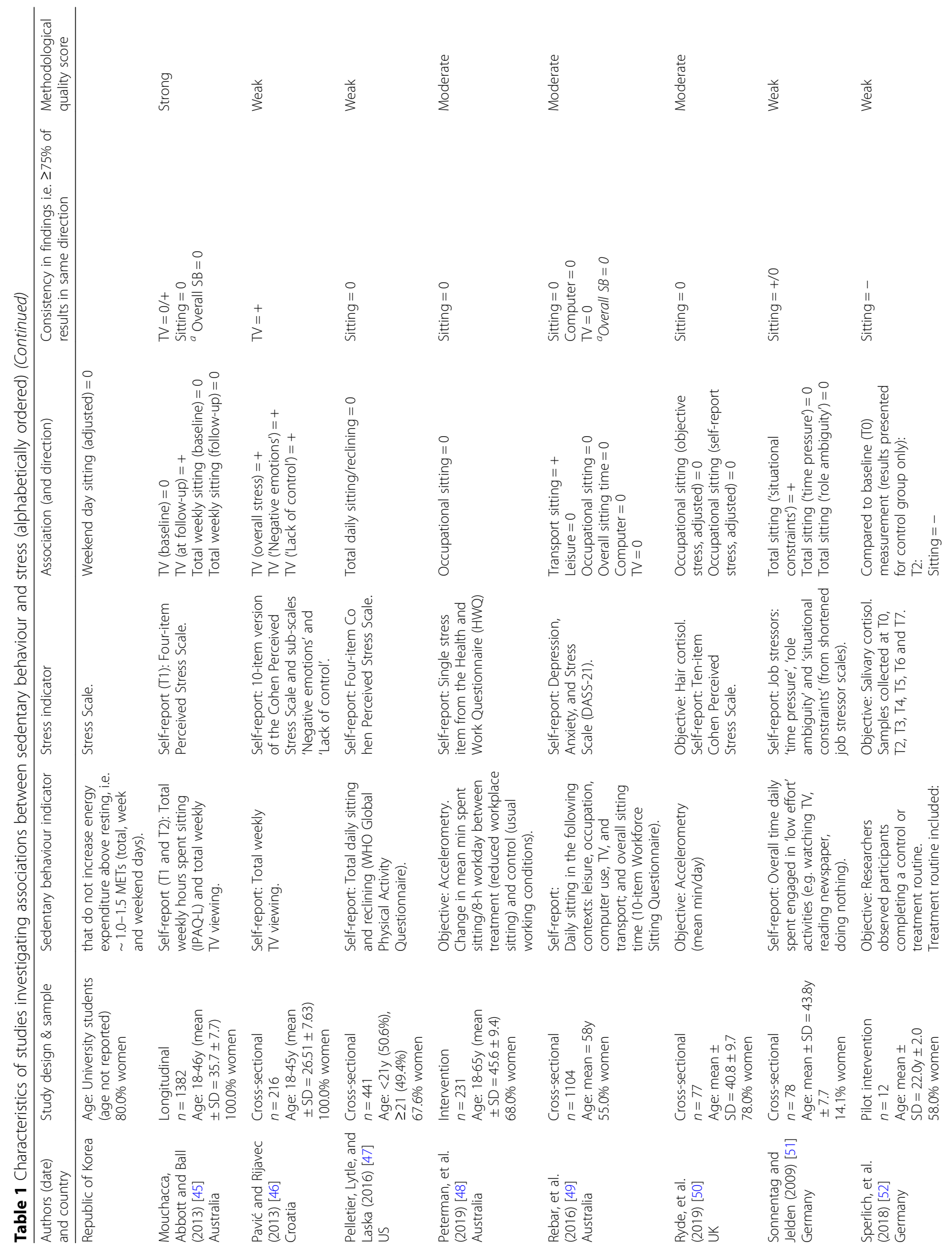




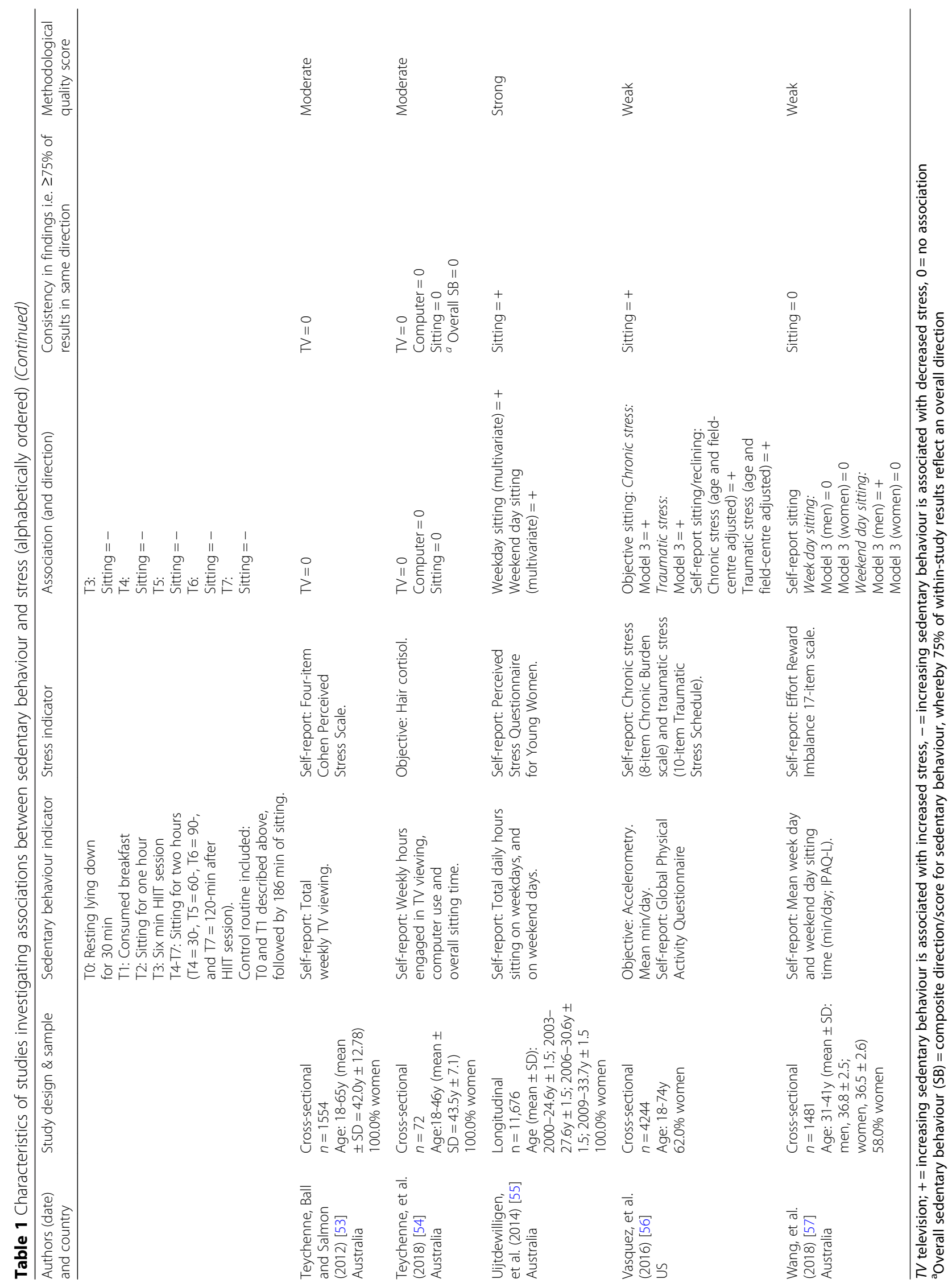


sitting time [49]. Reliability and validity of all the above measures of sedentary behaviour and stress are reported in Additional file 2: Table S2.

\section{Methodological quality}

Table 1 presents methodological quality scores. Overall, two studies were rated as strong [45, 55], 10 studies $[34,35,39,41,43,48-50,53,54]$ were rated as moderate and 14 studies [33, 36-38, 40, 44, 46, 47, 51, 52, 56, 57] received a weak methodological quality rating. Studies received weak or moderate ratings primarily due to study design limitations (e.g. 16 of 26 studies employed a crosssectional study design [33-36, 41, 43, 44, 46, 47, 49-51, 53, $54,56,57])$, participants were not likely to be representative of target population (8 of 26 studies) [37, 39, 40, 44, $46,47,51,52]$, or the studies did not employ/report reliable and/or valid measures for sedentary behaviour (11 of 26 studies) [33-36, 40, 44, 47, 51, 55] (see Additional file 2: Table S2).

Results are presented as a whole sample (i.e. combined findings) first, then for specific sedentary behaviours (i.e. overall sitting time, TV viewing, computer use), and finally for objectively versus subjectively assessed sedentary behaviour and stress.

\section{Combined findings}

Unadjusted results are reported here first, i.e. not adjusting for publication bias, nor for multiple modelling within studies. Overall, across the 26 studies, there were 78 models that assessed the association between sedentary behaviour (any indicator) and stress. This resulted in 29 positive associations (37\%; i.e. higher sedentary behaviour associated with higher levels of stress); eight inverse associations (10\%; i.e. higher sedentary behaviour associated with lower levels of stress) and 41 null results (53\%; i.e. no association between sedentary behaviour and stress).

Figure 2 provides a graphical representation (harvest plots) of the overall evidence. Specifically, of 26 studies reviewed, six ( $n=1$ strong quality [55]; $n=2$ moderatequality [34, 35]; $n=3$ methodologically weak-quality $[40,46,56])$ found a positive association between time spent in any sedentary behaviour and stress (i.e. increased time spent in sedentary behaviour was associated with increased stress). Five of these studies used self-report measures of stress [34, 35, 46, 55, 56], while one study [40] examined salivary cortisol. Eleven studies showed 'mixed' findings between sedentary behaviour and stress. Firstly, both positive and null associations were found among nine studies ( $\mathrm{n}=1$ strong- [45]; $n=2$ moderate- [41, 49]; $n=6$ weak-quality [33, 38, 44, 51, 57]). Stress was self-reported in eight of these studies [33, 38, $44,45,49,51,57]$, while one utilised objective measures (salivary cortisol) [41]).
Both positive and inverse associations between sedentary behaviour and self-reported stress were found in one weak-quality study [37], with the direction of these associations dependent on the source of stress assessed (e.g. total sitting was positively associated with 'argument-related' stress, but negatively associated with 'running late' stress) [37]. Two weak-quality studies which examined self-reported stress [36] and salivary cortisol [52] respectively, found inverse associations between time spent in sedentary behaviour and stress, whereby increased time in sedentary behaviour was associated with lower levels of stress. Eight remaining studies $(n=6 \bmod -$ erate- $[39,43,48,50,53,54] ; n=2$ weak-quality [33, 47]) found no association between sedentary behaviour and stress. Stress was self-reported in four of those studies [33, 47, 48, 53]; three used objective measures of stress (hair cortisol $[43,54]$; systolic and diastolic blood pressure, heart rate, and salivary cortisol [39]); whilst one study used both a self-report and objective (hair cortisol) measure [50].

\section{Best-evidence synthesis}

The best-evidence synthesis (adjusting for publication bias and multiple modelling within studies) resulted in the following: Excluding weak studies (since $\geq 2$ moderate/strong quality studies exist), three (25\%) ( $n=1$ strong- [55]; $n=2$ moderate-quality $[34,35])$ studies found a positive association between time spent in any sedentary behaviour and stress. One moderate-quality study (9\%) [41] showed 'mixed' (i.e. positive and null) findings and eight studies $(66 \%)(\mathrm{n}=1$ strong- [45]; $n=7$ moderate- [39, 43, 48-50, 53, 54] found predominately no association between sedentary behaviour and stress. Based on the best-evidence synthesis, there was insufficient evidence for an overall relationship between time spent in sedentary behaviour and stress.

\section{Overall sitting time}

Unadjusted results showed that across all studies, there were 60 models that assessed the association between sitting time and stress. This resulted in 23 positive associations (38\%); seven inverse associations (12\%) and 30 null results (50\%).

Specifically, a total of 19 studies $(n=2$ strong-quality $[45,55] ; n=8$ moderate-quality [34, 35, 39, 41, 48-50, 54]; $n=9$ weak-quality $[37,38,40,44,47,51,52,56,57])$ reported on associations between sitting time and stress. Five studies ( $n=1$ strong-quality [55]; $n=2$ moderatequality [34, 35]; $\mathrm{n}=2$ weak-quality $[40,56])$ found positive associations between sitting time and stress, i.e. increased sitting time was associated with increased stress. Of those, four employed self-reported measures of stress and nondomain-specific sitting measures, whilst one [40] utilised an objective measure of stress (i.e. salivary cortisol) and a 


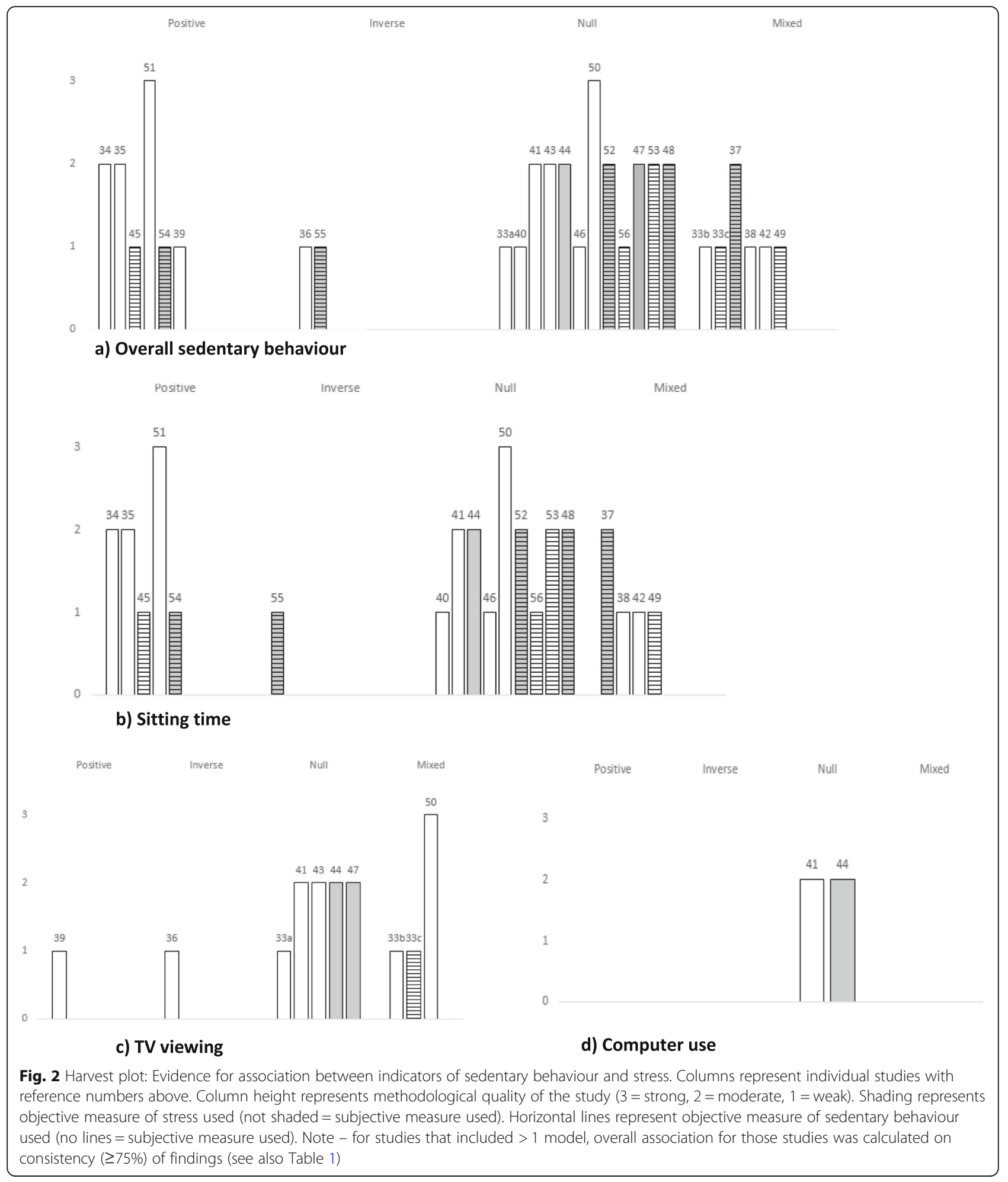

self-reported domain-specific measure of sitting (i.e. occupational sitting) .

One weak-quality study showed an inverse association between sitting time and objectively measured stress (i.e. salivary cortisol) [52]. Four studies ( $n=1$ moderate- [41]; $n=3$ weak-quality $[38,44,51])$ showed mixed findings. That is, they all included both positive and null results. For example, Lee \& Kim (2018) showed a positive association between total and weekday sitting time and stress, but no association between weekend sitting time 
and stress [44]. Gubelmann et al. (2018) showed a positive association between sitting and diurnal cortisol slope, but no association with mean or awakening cortisol [41]. Nine studies ( $n=1$ strong- [45]; $n=5$ moderate- $[39,48-50,54] ; n=3$ weak-quality [37, 47, 57]) showed predominately no association between sitting time and stress. Of note, however, are the findings from Rebar et al. (2016) [49]. That study compared sitting in three domains (transport-related, leisure-time, occupational) as well as overall sitting. Transport-related sitting time was positively associated with stress, whilst all other models (three of the four [i.e. $75 \%$ ]) showed no association (and hence the study was labelled as a null overall finding).

\section{Best-evidence synthesis}

Based on the inconsistent findings across studies (i.e. among the 10 moderate-strong quality studies included, three showed positive associations (30\%), one showed mixed (10\%), six showed null results $(60 \%))$, the bestevidence synthesis resulted in insufficient evidence for a relationship between overall sitting time and stress.

\section{TV viewing}

Unadjusted results showed that across all studies, there were 15 models that assessed the association between TV viewing and stress. This resulted in six positive associations (40\%); one inverse associations (7\%) and eight null associations $(53 \%)$.

Specifically, ten studies ( $n=1$ strong-quality [45]; $n=$ 4 moderate-quality $[43,49,53,54] ; n=5$ weak-quality $[33,36,46])$ investigated associations between TV viewing time and stress. One weak-quality study found a positive association between TV viewing and self-reported stress (i.e. increased TV viewing was associated with greater stress) [46]. Conversely, one weak-quality study found an inverse relationship between TV viewing and self-reported stress (i.e. increased TV viewing was associated with reduced stress) [36]. Mixed results, in this case positive and null associations, were found in two weak[33] and one strong- [45] quality study. For example, one longitudinal study found no cross-sectional association between TV viewing and perceived stress at baseline, but stress at baseline predicted increased TV viewing at follow-up [45]. Five ( $n=1$ weak-quality [33] and $n=4$ moderate-quality $[43,49,53,54])$ studies found no association between TV viewing and stress.

\section{Best-evidence synthesis}

Among the five moderate-strong quality studies that examined TV viewing, four (80\%) showed null associations and therefore based on the best-evidence synthesis there was strong evidence for no association between TV viewing and stress.

\section{Computer use}

Unadjusted results showed that across all studies, there were two models that assessed the association between computer use and stress, both of which found null associations (100\%). Specifically, two moderate-quality studies examined the association between computer use $[49,54]$ and stress. No association was found between computer use and self-reported stress [49], or computer use and objectively measured stress (hair cortisol) [54] in these studies $(100 \%)$. Based on the best-evidence synthesis there was strong evidence for no association between computer use and stress.

\section{Objectively measured versus self-reported stress}

Unadjusted results showed that across all studies, there were 18 models that assessed the association between sedentary behaviour and objectively measured stress. This resulted in two positive associations (11\%); six inverse associations (33\%) and ten null associations (56\%).

Specifically, of the seven studies that used an objective measure of stress $(n=2$ weak- $[40,52]$ and $n=5$ moderatequality [39, 41, 43, 50, 54]), two reported positive [40] or mixed (in this case positive and null) associations between sedentary behaviour and stress [41] (weak- and moderate quality studies, respectively). Four moderate-quality studies found null associations [39, 43, 50, 54], while one weakquality study showed inverse associations between sedentary behaviour and stress [52]. Based on the best-evidence synthesis (i.e. four of five moderate-quality studies showed null association (80\%), there was strong evidence for no association between any type of sedentary behaviour and objectively measured stress.

Unadjusted results showed that across all studies, there were 67 models that assessed the association between sedentary behaviour and self-reported stress. This resulted in 32 positive associations (48\%); eight inverse associations $(12 \%)$ and 27 null results $(40 \%)$.

Specifically, of the 20 studies that utilised self-report measures of stress, five studies $(n=1$ strong-quality [55]; $n=2$ moderate-quality [34, 35]; $n=2$ weakquality $[46,56])$ showed positive associations, while one weak-quality study showed inverse associations between sedentary behaviour and stress [36]. Five weak-quality studies showed 'mixed' (i.e. positive and null) associations between sedentary behaviour and stress [33, 38, 44, 51]). Nine other studies ( $n=1$ strong [45]; $n=4$ moderate- $[48-50,53]$; and $n=4$ weakquality [33, 37, 47, 57]) found no associations. Based on the best-evidence synthesis (i.e. three of eight (38\%) moderate-strong quality studies showed positive associations, five (62\%) showed null associations), there was insufficient evidence of a relationship between sedentary behaviour and self-reported stress. 


\section{Objectively measured versus self-reported sedentary behaviour}

Unadjusted results showed that across all studies, there were 39 models that assessed the association between objectively measured sedentary behaviour (i.e. device, direct observation) and stress. This resulted in 13 positive associations (33\%); seven inverse associations (18\%) and 19 null results (49\%).

Specifically, ten studies utilised objective measures of sedentary behaviour. Of those, two weak-quality studies showed positive associations [40,56]. Three studies showed 'mixed' (i.e. positive and null) associations between objectively measured sedentary behaviour and stress $(n=2$ weak[33, 38] and $n=1$ moderate-quality [41] studies). One weak-quality study found an inverse association [52]; while four studies ( $n=3$ moderate- $[39,48,50] ; n=1$ weakquality [37] found no associations. Based on the bestevidence synthesis (i.e. of the four moderate-quality studies, one (25\%) showed mixed, the other three (75\%) showed null associations), there was strong evidence for no relationship between objectively measured sedentary behaviour and stress.

Unadjusted results showed that across all studies, there were 39 models that assessed the association between self-reported sedentary behaviour and stress. This resulted in 16 positive associations (41\%); one inverse association (3\%) and 22 null results (56\%).

Specifically, studies that used self-report measures of sedentary behaviour $(n=16)$ yielded the following results. Four studies ( $n=1$ strong- [55]; $n=2$ moderate- [34, 35]; $n=1$ weak-quality [46]) found a positive association; four found 'mixed' associations (in this case, positive and null; $n=1$ strong- [45]; $n=3$ weak-quality [33, 44, 51]; one weak-quality study found an inverse association [36] and seven studies $(n=4$ moderate-quality [43, 49, 53, 54]; $\mathrm{n}=$ 3 weak- $[33,47,57])$ found no association between selfreported sedentary behaviour and stress. Based on the best-evidence synthesis (i.e. of the eight moderate-strong quality studies, three (37\%) showed positive, one (13\%) showed mixed, four (50\%) showed null associations) there was insufficient evidence for a positive relationship between self-reported sedentary behaviour and stress.

\section{Discussion}

To the best of our knowledge, this is the first review to systematically synthesise evidence of associations between time spent in sedentary behaviour and stress. Findings showed insufficient evidence for the association between overall sedentary behaviour, or sitting time and stress, particularly when using self-report measures of sedentary behaviour or stress. Further, we found strong evidence of no association between TV viewing, or computer use and stress; and strong evidence of no association between sedentary behaviour and stress when using objective measures of these outcomes.
Given that the findings between overall sitting time and stress were inconsistent, but TV viewing and computer use were strongly not associated with stress, this may indicate that the type of sedentary behaviour could potentially play a role in the link with stress. It is possible that time spent watching TV and using computers may have confounded the association between sitting time (which includes, but is not limited to, both of these behaviours) and stress. It may be that other sedentary behaviour indicators (e.g. smartphone and tablet use) that were not captured in these studies, yet contribute to overall sitting time, may be subsequently linked to greater stress. However, no studies utilised measures of these more modern electronic devices (i.e. smartphones/ tablets) and therefore this is a key area identified as requiring further research. It has been hypothesised that engaging in high levels of social media use (predominately undertaken whilst using modern electronic devices such as smartphones or tablets) may adversely impact mental health outcomes (e.g. anxiety, stress, depressive symptoms, including suicidal behaviours [23]), particularly since social media use has been associated with feelings of "addiction" and loss of sleep [58]. Further, it may be that watching TV or using computers (which are likely to be used for both leisure and occupational purposes) are not detrimental to mental health, given that some studies have suggested TV viewing may be used by adults experiencing depressive symptoms as a time to relax and 'switch off' [59].

Another finding of this review was that although there was insufficient evidence for the association between sedentary behaviour and stress when using self-report measures, there was strong evidence for no association between sedentary behaviour and stress when using objective measures of these outcomes. There is mixed evidence regarding the relationship between self-report and objectively-assessed stress. Whilst some studies have shown that self-report retrospective measures of stress are associated with objective measures (e.g. heart rate variability) of stress [60], other studies have shown that self-report measures of stress, assessed only contemporaneously but not retrospectively, are associated with objective measures (e.g. salivary cortisol) [61]. Although self-report measures can be subject to bias and recall error, objective measures also present limitations. For example, salivary cortisol levels fluctuate diurnally and can be influenced by other factors such as age or sleep [62]. Similarly, current objective, specifically device-assessed, measures of sedentary behaviour (e.g. inclinometers) are limited by the lack of distinction between the type (e.g. computer use, electronic device use, TV viewing) and context (e.g. work-related emails, social media use, online shopping) of activity being performed. Given that the type and/or context of sedentary behaviour could potentially be a key factor in the relationship with stress, rather 
than the sitting posture itself (as indicated by previous research examining associations between sedentary behaviour and other mental health outcomes [21, 22]), further studies utilising objective behaviour-specific measures of sedentary behaviour, which capture type and context, are required. This could include utilising existing technology such as smartphone apps (e.g. Moment app) that track the duration of time spent using electronic devices, which currently has not been done in studies examining the link between sedentary behaviour and mental health.

Only one study [49] examined associations between different domains of sitting time (i.e. transport, leisure, occupational) and stress, and results indicated some domains of sitting (in this case transport-related sitting) were more strongly associated with stress than other domains (i.e. leisure, occupational). These findings contrast those of another study that investigated the link between occupational sitting time and stress [40], which found a positive association. Although the domain of sedentary behaviour could potentially play a role in the relationship with stress, currently little research distinguishing domains of sedentary behaviour exists. This is another consideration when selecting measures for future studies, given that sitting at the computer for work purposes could potentially elicit differing stress responses compared to sitting at the computer for leisure purposes. To further add to the complexity of this research area, it has been suggested that standing (as opposed to sitting) whilst engaging in such screen-based behaviours (using a sit-stand workstation for example) may reduce the risk of poor mental health [63]. However, as previously described, the type and context of the sedentary behaviour is likely to be a key determinant in the relationship with stress, and is likely a more important determinant than the actual sitting/standing posture itself [49].

Studies included in this review were limited by other factors including: more than half $(54 \%)$ were rated as weak-quality and $62 \%$ utilised cross-sectional study designs in which temporal associations or cause and effect were unable to be determined. Studies used varied measures of sedentary behaviour and stress, which limits the ability to directly compare findings, and it was not possible to conduct a meta-analysis [29, 30]. A number of studies did not report on the reliability or validity of measures of sedentary behaviour and/or stress used. Further, there is a lack of evidence to suggest whether prolonged, uninterrupted sitting has a different effect or association on stress, compared to if one regularly breaks up their sitting time. In addition, no studies examined interactions between specific sedentary behaviours (e.g. TV viewing, computer use, electronic device use) and total sedentary time. Thus, it could be possible that the effects of, for example, TV viewing would be dependent on the total amount of sedentary behaviour (e.g., with a low total amount of sedentary behaviour the effects of TV viewing could be beneficial but with high total amount of sedentary behaviour the effects of TV viewing could be harmful). This is worthy of further examination.

\section{Conclusions}

Although limited (i.e. predominately weak-quality, crosssectional) research has explored associations between sedentary behaviour and stress, this review consolidates the existing evidence and found insufficient (due to conflicting results) evidence for the association between sedentary behaviour and overall sitting time and stress. Further there was strong evidence for no association between other specific types of sedentary behaviour (e.g. TV viewing, computer use) and stress. This review and the results presented provides preliminary information to question why we should investigate the link between sedentary behaviour and stress and highlights the importance of the activity that is being undertaken whilst the sitting is occurring (rather than the sitting itself). High-quality longitudinal/interventional research is required to confirm findings and determine the direction of associations between different types, contexts and domains of sedentary behaviour and stress.

\section{Supplementary information}

Supplementary information accompanies this paper at https://doi.org/10. 1186/s12889-019-7717-x.

Additional file 1: Table S1. Sedentary behaviour and stress systematic review databases and search terms

Additional file 2: Table S2. Methodological quality assessment of studies included in the present review

\section{Abbreviations}

CBS: Chronic Burden Scale; DASS: Depression, Anxiety and Stress Scale; DSI: Daily Stress Inventory; ERIS: Effort Reward Imbalance Scale; LEl: Life Events Inventory; PRISMA: Preferred reporting items for systematic reviews; PSQYW: Perceived Stress Questionnaire for Young Women; PSS: Cohen Perceived Stress Scale; SB: Sedentary behaviour; TSS: Traumatic Stress Schedule; TV: Television viewing; UK: United Kingdom; US: United States

\section{Acknowledgements \\ Not applicable}

\section{Authors' contributions}

MT, LDS, SAC, AIT contributed to the review's conception and design. LDS performed the systematic literature search; MT, LDS, and SAC analysed and interpreted data. MT and LDS led the writing of the manuscript. AIT, DLO and BS contributed to interpretation of the findings and drafting the manuscript. All authors read and approved the final manuscript for publication.

\section{Funding}

The study was supported in part by funding provided by the School of Exercise and Nutrition Sciences at Deakin University, of whom played no role in the design of the study and collection, analysis, and interpretation of data.

\section{Availability of data and materials}

The datasets supporting the conclusions of this article are included within the article (and Additional files). 


\section{Ethics approval and consent to participate}

Not applicable

\section{Consent for publication \\ Not applicable}

\section{Competing interests}

The authors declare that they have no competing interests.

\section{Author details}

'Institute for Physical Activity and Nutrition (IPAN), School of Exercise and Nutrition Sciences, Deakin University, Geelong, Australia. ${ }^{2}$ School of Exercise and Nutrition Sciences, Deakin University, Geelong, Australia. ${ }^{3}$ Department of Community Health Sciences, Cumming School of Medicine, University of Calgary, Calgary, Canada. ${ }^{4}$ Institute of Psychiatry, Psychology and Neuroscience, King's College London, London, UK.

\section{Received: 4 June 2019 Accepted: 30 September 2019}

\section{Published online: 23 October 2019}

\section{References}

1. Lazarus RS, Deese J, Osler SF. The effects of psychological stress upon performance. Psychol Bull. 1952;49(4):293.

2. Kalia M. Assessing the economic impact of stress [mdash] the modern day hidden epidemic. Metabolism. 2002;51(6):49-53.

3. McEwen BS. Stressed or stressed out: what is the difference? J Psychiatry Neurosci. 2005;30(5):315-8.

4. Swedish National Institute of Public Health: Folkhälsorapport 2009 Stockholm. Stockholm, Sweden:: Socialstyrelsen; 2009.

5. Australian Psychological Society: Australian Psychological Society Stress and wellbeing in Australia survey 2015. 2015.

6. Work. EAfSaHa: European Risk Observatory Report: OSH in figures: stress at work - facts and figures. Luxembourg: European Communities; 2009.

7. American Psychological Association. Stress in America: The impact of discrimination, Stress in America Survey. 2016. https://www.apa.org/news/ press/releases/stress/2015/impact-of-discrimination.pdf. Accessed 13 Oct 2019.

8. Steptoe A, Kivimäki M. Stress and cardiovascular disease. Nat Rev Cardiol. 2012;9:360.

9. Mazure CM. Life stressors as risk factors in depression. Clin Psychol. 1998; 5(3):291-313.

10. Rai D, Kosidou K, Lundberg M, Araya R, Lewis G, Magnusson C. Psychological distress and risk of long-term disability: population-based longitudinal study. J Epidemiol Community Health. 2012;66(7):586-92.

11. Toppinen-Tanner S, Ojajärvi A, Väänaänen A, Kalimo R, Jäppinen P. Burnout as a predictor of medically certified sick-leave absences and their diagnosed causes. Behav Med. 2005;31(1):18-32.

12. Hassard J, Teoh KRH, Visockaite G, Dewe P, Cox T. The cost of work-related stress to society: a systematic review. J Occup Health Psychol. 2018;23(1):1-17.

13. Medibank Private: The cost of workplace stress in Australia. Medibank Private Limited; 2008

14. Salmon P. Effects of physical exercise on anxiety, depression, and sensitivity to stress: a unifying theory. Clin Psychol Rev. 2001;21(1):33-61.

15. Wilcox S, Dowda M, Leviton LC, Bartlett-Prescott J, Bazzarre T, CampbellVoytal K, Carpenter RA, Castro CM, Dowdy D, Dunn AL, Griffin SF, Guerra M, King AC, Ory MG, Rheaume C, Tobnick J, Wegley S. Active for life: final results from the translation of two physical activity programs. Am J Prev Med. 2008;35(4):340-51.

16. Gonzalez MJ, Miranda-Massari JR. Diet and stress. Psychiatr Clin. 2014: 37(4):579-89.

17. Taylor G, McNeill A, Girling A, Farley A, Lindson-Hawley N, Aveyard P. Change in mental health after smoking cessation: systematic review and meta-analysis. Bmj. 2014;348:g1151.

18. Molendijk M, Molero P, Sánchez-Pedreño FO, Van der Does W, MartínezGonzález MA. Diet quality and depression risk: a systematic review and dose-response meta-analysis of prospective studies. J Affect Disord. 2017

19. Rebar AL, Stanton R, Geard D, Short C, Duncan MJ, Vandelanotte C. A metameta-analysis of the effect of physical activity on depression and anxiety in non-clinical adult populations. Health Psychol Rev. 2015;9(3):366-78.

20. Tremblay MS, Aubert S, Barnes JD, Saunders TJ, Carson V, Latimer-Cheung AE, Chastin SFM, Altenburg TM, Chinapaw MJM, Participants STCP.
Sedentary behavior research network (SBRN) - terminology consensus project process and outcome. Int J Behav Nutr Phys Act. 2017:14(1):75.

21. Teychenne M, Ball K, Salmon J. Sedentary behavior and depression among adults: a review. Int J Behav Med. 2010;17(4):246-54.

22. Teychenne M, Costigan SA, Parker K. The association between sedentary behaviour and risk of anxiety: a systematic review. BMC Public Health. 2015; 15:513.

23. Cheng Y-S, Tseng P-T, Lin P-Y, Chen T-Y, Stubbs B, Carvalho AF, Wu C-K, Chen Y-W, Wu M-K. Internet Addiction and Its Relationship With Suicidal Behaviors: A Meta-Analysis of Multinational Observational Studies. J Clin Psychiatry. 2018:79(4). https://doi.org/10.4088/JCP.17r11761.

24. Fossum IN, Nordnes LT, Storemark SS, Bjorvatn B, Pallesen S. The association between use of electronic media in bed before going to sleep and insomnia symptoms, daytime sleepiness, morningness, and chronotype. Behav Sleep Med. 2014;12(5):343-57.

25. Teychenne M, Hinkley T. Associations between screen-based sedentary behaviour and anxiety symptoms in mothers with young children. PLoS One. 2016;11(5):e0155696.

26. Moher D, Liberati A, Tetzlaff J, Altman DG, Group P. Preferred reporting items for systematic reviews and meta-analyses: the PRISMA statement. PLoS Med. 2009;6(7):e1000097.

27. Taintor Z, Rosenthal RN. Microprocessor abuse and internet addiction. In: Ries RK, Fiellin DA, Miller SC, Saitz R, editors. Principles of Addiction Medicine. 4th ed. Philadelphia: Lippincott, Williams and Wilkins; 2009.

28. National Collaborating Centre for Methods and Tools. Quality assessment tool for quantitative studies. Hamilton: McMaster University; 2008. Retrieved from http://www.nccmt.ca/resources/ search/14. Accessed 03 Oct 2017

29. Slavin RE. Best-evidence synthesis: An alternative to Meta-analytic and traditional reviews. Educ Res. 1986;15(9):5-11.

30. Cochrane Effective Practice and Organisation of Care (EPOC). Synthesising results when it does not make sense to do a meta analysis. EPOC Resources for review authors 20172017 [https://epoc. cochrane.org/sites/epoc.cochrane.org/files/public/uploads/Resources-forauthors2017/synthesising_results_when_meta-analysis_does_not_make_ sense.pdf ] (accessed: 1 April 2019).

31. Proper Kl, Singh AS, van Mechelen W, Chinapaw MJ. Sedentary behaviors and health outcomes among adults: a systematic review of prospective studies. Am J Prev Med. 2011:40(2):174-82.

32. Chinapaw MJ, Proper Kl, Brug J, van Mechelen W, Singh AS Relationship between young peoples' sedentary behaviour and biomedical health indicators: a systematic review of prospective studies. Obes Rev. 2011;12(7):e621-32.

33. Anderson DR, Collins PA, Schmitt KL, Jacobvitz RS. Stressful life events and television viewing. Commun Res. 1996;23(3):243-60.

34. An KO, Jang JY, Kim J. Sedentary behavior and sleep duration are associated with both stress symptoms and suicidal thoughts in korean adults. Tohoku Exp Med. 2015:237(4):279-86.

35. Ashdown-Franks G, Koyanagi A, Vancampfort D, Smith L, Firth J, Schuch F, Veronese N, Stubbs B. Sedentary behavior and perceived stress among adults aged $\geq 50$ years in six low- and middle-income countries. Maturitas. 2018;116:100-7.

36. Depp CA, Schkade DA, Thompson WK, Jeste DV, Depp CA, Schkade DA, Thompson WK, Jeste DV. Age, affective experience, and television use. Am J Prev Med. 2010;39(2):173-8.

37. Diaz KM, Thanataveerat A, Parsons FE, Yoon S, Cheung YK, Alcántara C, Duran AT, Ensari I, Krupka DJ, Schwartz JE, Burg MM, Davidson KW. The influence of daily stress on sedentary behavior: group and person ( $\mathrm{N}$ of 1) level results of a 1-year observational study. Psychosom Med. 2018; 80(7):620-7.

38. Ellingson LD, Meyer JD, Shook RP, Dixon PM, Hand GA, Wirth MD, Paluch AE, Burgess S, Hebert JR, Blair SN. Changes in sedentary time are associated with changes in mental wellbeing over 1 year in young adults. Prev Med Rep. 2018;11:274-81

39. Endrighi R, Steptoe A, Hamer M. The effect of experimentally induced sedentariness on mood and psychobiological responses to mental stress. $\mathrm{Br}$ J Psychiatry. 2016:208(3):245-51.

40. Gilson ND, Hall C, Renton A, Ng N, von Hippel W. Do sitting, standing, or treadmill desks impact psychobiological indicators of work productivity? J Phys Act Health. 2017;14(10):793-6. 
41. Gubelmann C, Kuehner C, Vollenweider P, Marques-Vidal P. Association of activity status and patterns with salivary cortisol: the population-based CoLaus study. Eur J Appl Physiol. 2018.

42. Adam EK, Kumari M. Assessing salivary cortisol in large-scale, epidemiological research. Psychoneuroendocrinology. 2009;34(10):1423-36

43. Jackson SE, Firth J, Grabovac I, Koyanagi A, Stubbs B, Soysal P, Willmott A, Yang L, Smith L. Sedentary behaviour and chronic stress in old age: a crosssectional analysis of TV viewing and hair cortisol concentrations. Psychoneuroendocrinology. 2019;109:104375.

44. Lee E, Kim Y. Effect of university students' sedentary behavior on stress, anxiety, and depression. Perspect Psychiatr Care. 2018.

45. Mouchacca J, Abbott GR, Ball K. Associations between psychological stress, eating, physical activity, sedentary behaviours and body weight among women: a longitudinal study. BMC Public Health. 2013;13(1):828.

46. Pavić J, Rijavec M. Stress and television viewing in female college students: mediating role of TV viewing motives and TV affinity. Suvremena Psihologija. 2013;16(1):33-47.

47. Pelletier JE, Lytle LA, Laska MN. Stress, health risk behaviors, and weight status among community college students. Health Educ Behav. 2016; 43(2):139-44.

48. Peterman JE, Healy GN, Winkler EA, Moodie M, Eakin EG, Lawler SP, Owen N, Dunstan DW, LaMontagne AD. A cluster randomized controlled trial to reduce office workers' sitting time: effect on productivity outcomes. Scand J Work Environ Health. 2019;45(5):483-92.

49. Rebar AL, Vandelanotte C, Van Uffelen J, Short C, Duncan MJ. Associations of overall sitting time and sitting time in different contexts with depression, anxiety, and stress symptoms. Ment Health Phys Act. 2016;7(2):105-10.

50. Ryde GC, Dreczkowski G, Gallagher I, Chesham R, Gorely T. Device-Measured Desk-Based Occupational Sitting Patterns and Stress (Hair Cortisol and Perceived Stress). Int J Environ Res Public Health. 2019;16(11). https://doi. org/10.3390/ijerph16111906.

51. Sonnentag S, Jelden S. Job stressors and the pursuit of sport activities: a day-level perspective. J Occup Health Psychol. 2009;14(2):165-81.

52. Sperlich B, De Clerck I, Zinner C, Holmberg HC, Wallmann-Sperlich B: Prolonged sitting interrupted by 6 -min of high-intensity exercise: Circulatory, metabolic, hormonal, thermal, cognitive, and perceptual responses. Frontiers in Physiology 2018, 9(OCT).

53. Teychenne M, Ball K, Salmon J. Correlates of socio-economic inequalities in women's television viewing: a study of intrapersonal, social and environmental mediators. Int J Behav Nutr Phys Act. 2012;3.

54. Teychenne M, Olstad DL, Turner Al, Costigan SA, Ball K: Sedentary Behaviour and Hair Cortisol Amongst Women Living in Socioeconomically Disadvantaged Neighbourhoods: A Cross-Sectional Study. Int J Environ Res Public Health 2018, 15(4) doi: https://doi.org/10.3390/ijerph15040586.

55. Uijtdewilligen L, Twisk JWR, Singh AS, Chinapaw MJM, van Mechelen W, Brown WJ. Biological, socio-demographic, work and lifestyle determinants of sitting in young adult women: A prospective cohort study. Int J Behav Nutr Phys Act. 2014;11(1):7.

56. Vasquez E, Strizich G, Gallo L, Marshall SJ, Merchant GC, Murillo R, Penedo FJ, Salazar C, Sotres-Alvarez D, Shaw BA, Isasi CR. The role of stress in understanding differences in sedentary behavior in Hispanic/Latino adults: results from the Hispanic community health study/study of Latinos sociocultural ancillary study. J Phys Act Health. 2016;13(3):310-7.

57. Wang S, Sanderson K, Dwyer T, Venn A, Gall S. Job stress in young adults is associated with a range of poorer health behaviors in the childhood determinants of adult health (CDAH) study. J Occup Environ Med. 2018;60(3):e117-25.

58. Lougheed E. razzled by Facebook? An exploratory study of gender differences in social network communication among undergraduate men and women. Coll Stud J. 2012:88-99.

59. Teychenne M, Ball K, Salmon J. Perceived influences on and strategies to reduce sedentary behavior in disadvantaged women experiencing depressive symptoms: a qualitative study. Ment Health Phys Act. 2011;4:95-102.

60. Föhr T, Tolvanen A, Myllymäki T, Järvelä-Reijonen E, Rantala S, Korpela R, Peuhkuri K, Kolehmainen M, Puttonen S, Lappalainen R. Subjective stress, objective heart rate variability-based stress, and recovery on workdays among overweight and psychologically distressed individuals: a crosssectional study. J Occup Med Toxicol. 2015;10(1):39.

61. Metzenthin P, Helfricht S, Loerbroks A, Terris DD, Haug HJ, Subramanian $\mathrm{S}$, Fischer JE. A one-item subjective work stress assessment tool is associated with cortisol secretion levels in critical care nurses. Prev Med. 2009;48(5):462-6.
62. Ice GH. Factors influencing cortisol level and slope among community dwelling older adults in Minnesota. J Cross Cult Gerontol. 2005;20(2):91.

63. Pronk NP, Katz AS, Lowry M, Payfer JR. Peer reviewed: reducing occupational sitting time and improving worker health: the take-a-stand project, 2011. Prev Chronic Dis. 2012:9.

\section{Publisher's Note}

Springer Nature remains neutral with regard to jurisdictional claims in published maps and institutional affiliations.
Ready to submit your research? Choose BMC and benefit from:

- fast, convenient online submission

- thorough peer review by experienced researchers in your field

- rapid publication on acceptance

- support for research data, including large and complex data types

- gold Open Access which fosters wider collaboration and increased citations

- maximum visibility for your research: over $100 \mathrm{M}$ website views per year

At $\mathrm{BMC}$, research is always in progress.

Learn more biomedcentral.com/submissions 